\title{
Leishmania infection and blood sources analysis in Phlebotomus chinensis (Diptera: Psychodidae) along extension region of the loess plateau, China
}

Han-Ming Chen ${ }^{1}$, Hui-Ying Chen ${ }^{1}$, Feng Tao ${ }^{1}$, Jing-Peng Gao ${ }^{1}$, Kai-Li Li ${ }^{1}$, Hua Shi ${ }^{2}$, Heng Peng ${ }^{3^{*}}$ and Ya-Jun Ma ${ }^{1 *}$ (D)

\begin{abstract}
Background: Visceral leishmaniasis (VL) was one of the most important parasitic diseases in China, caused by Leishmania protozoans and transmitted by sand flies. Recently VL cases have reappeared in China, including the extension region of the Loess Plateau. The purpose of this study was to collect fundamental data on the hostvector VL system in the Loess Plateau to assist in the development of prevention and control measures.

Methods: Sand flies were collected by light traps from rural areas in Shanxian, Henan, China in 2015, as well as in Wuxiang and Yangquan, Shanxi, China in 2017. The blood sources of sand flies were analyzed by PCR detecting the host-specific mitochondrial cytochrome $b$ (mtDNA cyt b) gene fragments. Leishmania infection in sand flies was detected by amplifying and sequencing ribosomal DNA internal transcribed spacer 1 (ITS1). The Leishmania specific antibodies in the sera of local dogs were detected by ELISA kit.

Results: Blood sources showed diversity in the extension region of the Loess Plateau, including human, chicken, dog, cattle, pig and goat. Multiple blood sources within a sand fly were observed in samples from Yangquan (17/ $118,14.4 \%)$ and Wuxiang (12/108, 11.1\%). Leishmania DNA was detected in sand flies collected from Yangquan with minimum infection rate of $1.00 \%$. The ITS1 sequences were conserved with the Leishmania donovani complex. The positive rate of Leishmania specific antibodies in dogs was 5.97\%.

Conclusions: This study detected the blood sources and Leishmania parasites infection of sand flies by molecular methods in the extension region of Loess Plateau, China. A high epidemic risk of leishmaniasis is currently indicated by the results as the infection of Leishmania in sand flies, the extensive blood sources of sand flies including humans, and positive antibody of Leishmania in local dog sera. Given the recent increase of VL cases, asymptomatic patients, dogs and other potential infected animals should be screened and treated. Furthermore, the density of sand flies needs to be controlled and personal protection should be strengthened.
\end{abstract}

Keywords: Sand fly, Leishmania, Blood source, Extension region of the loess plateau, China

\footnotetext{
*Correspondence: pengheng0923@126.com; yajun_ma@163.com

${ }^{3}$ Department of Medical Microbiology and Parasitology, College of Basic

Medical Sciences, Naval Medical University, 800 Xiangyin Road, Shanghai

200433, China

'Department of Naval Medicine, Naval Medical University, 800 Xiangyin Road,

Shanghai 200433, China

Full list of author information is available at the end of the article
}

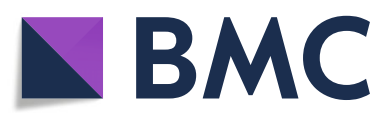

(- The Author(s). 2020 Open Access This article is licensed under a Creative Commons Attribution 4.0 International License, which permits use, sharing, adaptation, distribution and reproduction in any medium or format, as long as you give appropriate credit to the original author(s) and the source, provide a link to the Creative Commons licence, and indicate if changes were made. The images or other third party material in this article are included in the article's Creative Commons licence, unless indicated otherwise in a credit line to the material. If material is not included in the article's Creative Commons licence and your intended use is not permitted by statutory regulation or exceeds the permitted use, you will need to obtain permission directly from the copyright holder. To view a copy of this licence, visit http://creativecommons.org/licenses/by/4.0/. The Creative Commons Public Domain Dedication waiver (http://creativecommons.org/publicdomain/zero/1.0/) applies to the data made available in this article, unless otherwise stated in a credit line to the data. 


\section{Background}

Visceral leishmaniasis (VL), also known as kala-azar, is a sand fly borne disease caused by Leishmania protozoans. VL was one of the most important parasitic diseases in China [1-4]. At present, VL is mainly endemic in western China, and cases occurred in Xinjiang, Inner Mongolia, Gansu, Sichuan, Shaanxi, and Shanxi [2, 3]. From 2004 to 2012, a total of 3337 cases were reported, of which $97.03 \%$ were distributed in Xinjiang, Gansu and Sichuan [5]. In 2018, 180 cases of VL were reported in 78 counties of 11 provinces in China, mainly distributed in Gansu, Shanxi and Shaanxi Province, and the endemic area has expanded [6], indicating the rising risk of VL in China.

Three endemic types of VL have been described in China, the anthroponotic type (AVL), the zoonotic desert type (DT-ZVL) and zoonotic mountain type (MTZVL) [7]. AVL was endemic in the Kashgar alluvial plain and the Aksu oasis of Xinjiang, and it was historically called "anthroponotic" because no reservoir animal was ever found. DT-ZVL is dominant in ancient oases and deserts in Xinjiang and Inner Mongolia, and although in some places it, too, has an unknown reservoir, the Tarim hare (Lepus yarkandensis) has been identified as its reservoir in some endemic areas. The extension region of Loess Plateau in Shanxi, Henan and Shaanxi Provinces was one of the typical MT-ZVL endemic regions. The characteristics of the MT-ZVL include: the pathogen was mainly Leishmania infantum, the vector was Phlebotomus chinensis, and the zoonotic hosts were dog (Canis familiaris) as well as raccoon dog (Nyctereutes procyonoides) [8-11].

In China, VL was eliminated in most endemic areas after stringent implementation of control programs by the government in the 1950s [8]. However, local VL cases began to reappear since the early twenty-first century [12, 13]. In 2018, $38 \mathrm{VL}$ cases were reported in Shanxi Province, mainly in Yangquan City (15 cases) and Pingding County (8 cases). In addition, three and 27 cases were reported in Henan and Shaanxi provinces [6].

The reason of VL recurrence in the extension region of Loess Plateau was puzzled, which was an obstacle to develop prevention and control measures. Blood meal identification is important evidence for the determination of the host preferences of hematophagous arthropods. Detection of Leishmania infection in sand flies and animal hosts could provide critical information to estimate the vector competence and assess the epidemic risk of VL in the endemic areas [14, 15]. However, there was no report on Leishmania infection and blood source animals of sand flies in the extension region of Loess Plateau.

In order to explore the reasons for the VL recurrence in the area, we collected sand flies from three sites in the extension region of the Loess Plateau, China including Shanxian in Henan Province, Yangquan and Wuxiang in
Shanxi Province. Leishmania infection and blood sources were detected, and the Leishmania specific antibodies were determined in the sera of local dogs.

\section{Materials and methods}

Ethics statement

This study was carried out in strict accordance with the National Natural Science Foundation of China ethical guidelines for biomedical research involving living animals and human subjects (2016).

\section{Sand fly collection and species identification}

The sand fly samples were collected at three sites located in the extension region of the Loess Plateau, China (Table 1, Fig. 1): Xizhang Village $\left(111.22^{\circ} \mathrm{E} / 34.62^{\circ} \mathrm{N}, 985\right.$ $\mathrm{m})$ in Shanxian County (SX), Henan Province in July 2015, Hedi Village $\left(113.56^{\circ} \mathrm{E} / 38.00^{\circ} \mathrm{N}, 895 \mathrm{~m}\right)$ in Yangquan City (YQ) and Moyu Village $\left(113.09^{\circ} \mathrm{E} / 36.79^{\circ} \mathrm{N}\right.$, $1050 \mathrm{~m}$ ) in Wuxiang County (WX), Shanxi Province, China in June 2017.

Light traps (MYFS-HJY-1, Houji Dianzi, Dongguan, China) were used to catch sand flies. With the consent of the owners, light traps were set up in utility room, cave dwelling, courtyard and chicken farm from 5:30 pm to $8: 30 \mathrm{am}$, and collected manually in the evening by mouth aspirators. The captured sand flies were sorted and counted by male and female, respectively.

Some fresh female sand fly adults were randomly selected to dissect to check our molecular identifications. The species were identified in according with the morphology of pharyngeal armature and spermatheca [16]. The rest of the specimens were preserved in RNAfixer (Aidlab Biotechnologies, China) and brought back to the laboratory. All samples used in this study were identified by DNA sequences. Blood source analysis was conducted on those female specimens with visible blood residues, whereas all female sand flies were used to detect Leishmania infection.

\section{The ecological niches of the sand flies}

The ecological niches of the sand flies in the extension region of the Loess Plateau China were described in our published article [17]. In brief, the collection sites are located in hilly lands with altitude ranges from $895 \mathrm{~m}$ to $1050 \mathrm{~m}$, with similar geographical features and typical northern temperate climate. The buildings are cave dwellings or brick houses with tile roof. There are a variety of domesticated animals in the villages, including chickens, dogs, pigs, cattle and goats. Most animals were kept in caves or semi-closed livestock circles adjacent to the houses, and some animals are kept open in the courtyard. 
Table 1 The information on sample collection of sandflies in the extension of the Loess Plateau, China

\begin{tabular}{llllll}
\hline Collection site & & Date & Longitude & Latitude & Altitude (m) \\
\hline Xizhang Village, Shanxian County, Henan Province, China & $\begin{array}{l}\text { Chicken sheds, cave dwelling and } \\
\text { (SX) }\end{array}$ & July 2015 & $111.22^{\circ} \mathrm{E}$ & $34.62^{\circ} \mathrm{N}$ & 985 \\
Courtyard & & & & \\
Hedi Village, Yangquan City, Shanxi Province, China (YQ) & Chicken farm, utility room and courtyard & June 2017 & $113.56^{\circ} \mathrm{E}$ & $38.00^{\circ} \mathrm{N}$ & 895 \\
Moyu Village, Wuxiang County, Shanxi Province, China (WX) & Livestock sheds & June 2017 & $113.09^{\circ} \mathrm{E}$ & $36.79^{\circ} \mathrm{N}$ & 1050 \\
\hline
\end{tabular}

\section{DNA extraction and molecular identification of sand flies species}

Genomic DNA of sand fly samples was extracted using DNAzol (Life Technologies, USA) following the manufacturer's instructions. The fragment of the mitochondrial cytochrome $b$ (mtDNA cyt $b$ ) gene of sand flies was amplified according to the method reported by Esseghir [18]. The primers were forward CB1 (5'-TAT GTA CTA CCA TGA GGA CAA ATA TC-3') and reverse CB3-R3A (5'-GCT AAT TAC TCC TCC TAA CTT ATT-3'). The positive PCR products were sequenced using four-color fluorescently labeled dideoxy termination method in Boshang Biotech Co., Ltd. (Shanghai, China). The sequences were Blast aligned in GenBank on the NCBI website (https://blast.ncbi.nlm.nih. gov/Blast.cgi) to determine the sand fly species.

\section{Blood sources identification}

The female sand flies with visible blood residues were used for blood sources analysis, including 31 pooled and
226 individual samples. There were 12 pools from SX, 9 pools from YQ and 10 pools from WX. Every pooled sample contained 10 individuals. The individual samples include 118 from YQ and 108 from WX. The mtDNA cyt $b$ fragments of different animals and human were amplified by PCR [19-22]. According to the main animal species in the collection site, PCR assay was developed with primers specific to human, chicken, goat, pig, cattle and dog. The information of primer sets was listed in Table 2, and separate PCRs were performed for each pair of primers. The PCR reaction was carried out in $25 \mu \mathrm{l}$ containing $1.5 \mu \mathrm{l}$ DNA template, $0.2 \mu \mathrm{mol} / \mathrm{L}$ primers and $12.5 \mu \mathrm{l} 2 \times$ PCR mix reagents (Aidlab Biotechnologies, China). The PCR running parameters were starting at $94^{\circ} \mathrm{C}$ for $2 \mathrm{~min}$; continuing with 35 cycles of $94^{\circ} \mathrm{C}$ for $15 \mathrm{~s}, 51^{\circ} \mathrm{C}$ for $30 \mathrm{~s}$, and $72{ }^{\circ} \mathrm{C}$ for $1 \mathrm{~min}$; and a final extension with $72{ }^{\circ} \mathrm{C}$ for $8 \mathrm{~min}$. The PCR products were electrophoresed on a $1.5 \%$ agarose gel to determine the size and were sequenced to confirm.

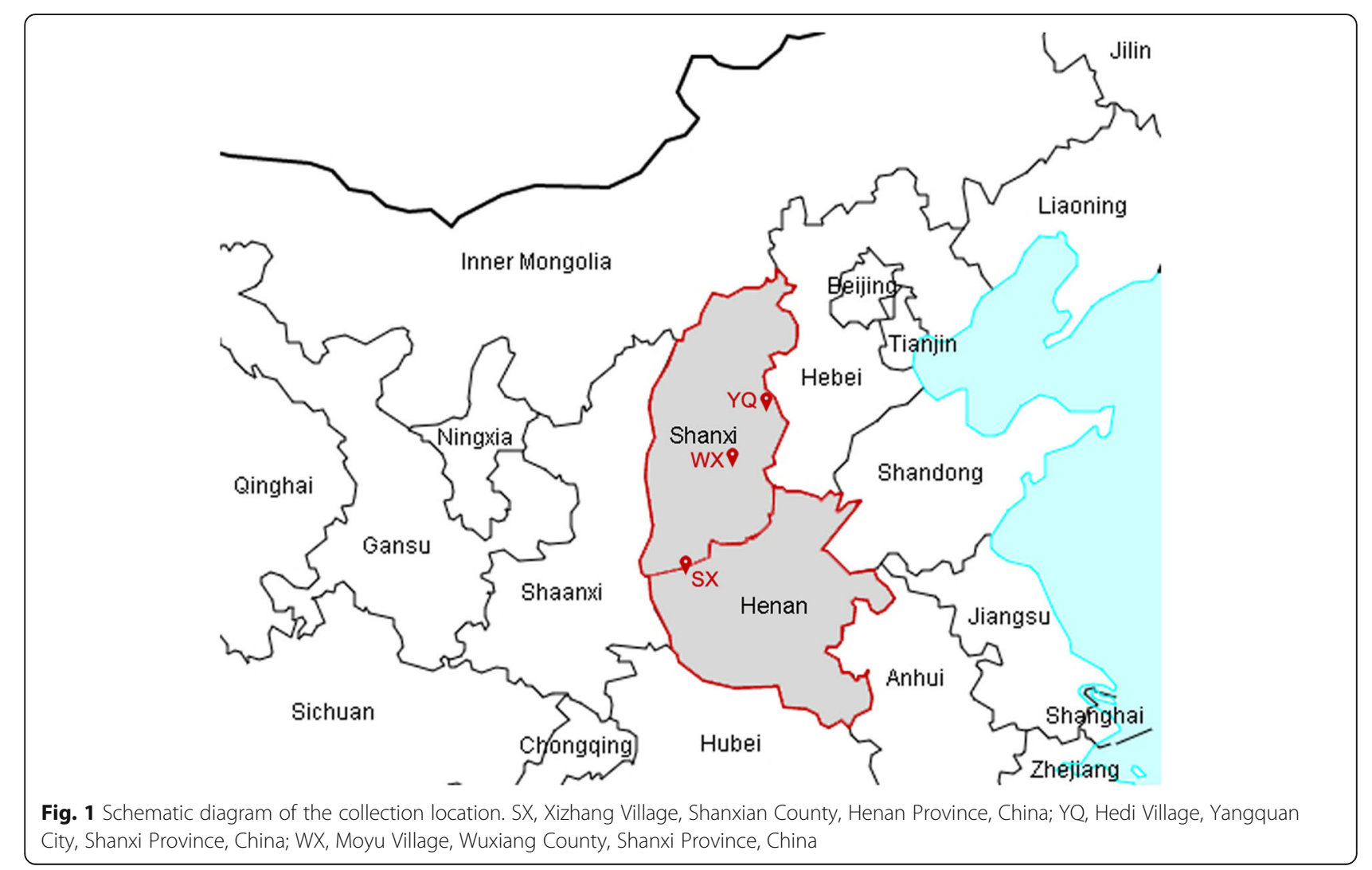


Table 2 PCR primers for mtDNA-cytb amplification of blood sources of sandflies

\begin{tabular}{lllc}
\hline Species & Primer & Sequence $\left(5^{\prime} \rightarrow 3^{\prime}\right)$ & Amplicon length (bp) \\
\hline Human (Homo sapiens) & HF & GGC TTA CTT CTC TTC ATT CTC TCC T & 334 \\
Chicken (Gallus gallus) & GR & GGT TGT CCT CCA ATT CAT GTT A & \\
Goat (Capra hircus) & CAT ACT CCC TCA CTC CCC CA & CCC CTC AGG CTC ACT CTA CT & 802 \\
Pig (Sus domesticus) & GoatF & AAT CAT CCG ATA CAT ACA CG \\
& GoatR & ATA TAG TTG TCT GGG TCT CC \\
Cattle (Bos taurus) & CCT CGC AGC CGT ACA TCT C & GGT TGT CCT CCA ATT CAT GTT A \\
Dog (Canis familiaris) & CAT CGG CAC AAA TTT AGT CG & 506 \\
& GF & GGT TGT CCT CCA ATT CAT GTT A & 563 \\
\hline
\end{tabular}

\section{Leishmania spp. detection in sand flies}

Leishmania infection was identified in all female sand flies. The ribosomal DNA internal transcribed spacer 1 (ITS 1) fragment of Leishmania was amplified using the following primers: forward LITSR 5'-CTG GAT CAT TTT CCG ATG-3', reverse L5.8S 5'-TGA TAC CAC TCG CAC TT-3' [23]. The PCR reaction was performed in $25 \mu \mathrm{l}$ contained $1.5 \mu \mathrm{l}$ DNA template, $0.2 \mu \mathrm{mol} / \mathrm{L}$ primers and $12.5 \mu \mathrm{l} 2 \times \mathrm{PCR}$ mix reagents. The PCR temperature profile was as follows: starting at $94{ }^{\circ} \mathrm{C}$ for $2 \mathrm{~min}$; continuing with 35 cycles of $94^{\circ} \mathrm{C}$ for $30 \mathrm{~s}, 52^{\circ} \mathrm{C}$ for $30 \mathrm{~s}$, and $72{ }^{\circ} \mathrm{C}$ for $30 \mathrm{~s}$; and a final extension with $72{ }^{\circ} \mathrm{C}$ for $8 \mathrm{~min}$. A positive control containing Leishmania donovani DNA (provided by National Institute of Parasitic Diseases, Chinese Center for Disease Control and Prevention) and a negative control without DNA template were utilized.

Detection of anti-Leishmania antibody in the sera of dogs The sera of 67 dogs (Canis familiaris) from YQ were provided by Yangquan Center for Disease Control and Prevention. The dogs were $0.5-14$ years old. The owners of the dogs had been informed and consented to the blood collection. The sera were separated by centrifugation at $1000 \times g$ for $10 \mathrm{~min}$ after blood collection in the field. Then the sera were refrigerated and transported to the laboratory for testing. The Leishmania specific antibodies were detected using the commercialized Dogs Leishmania Ab ELISA kit (Fusheng Industrial Co., Ltd. Shanghai, China). A 96-well ELISA plate coated with Leishmania antigen, positive and negative control sera were provided in the kit. The serum samples were diluted and assayed according to the manufacturers' instructions. Briefly, diluted serum samples, positive and negative control sera were added to the 96-well ELISA plate coated with antigen. After incubation and subsequent washing, the HRP-conjugate antigen was added to the wells. The plate was washed again after incubation. Then the HRP enzyme substrate was added for chromogenic reaction. The optical density (OD) value was recorded at $450 \mathrm{~nm}$ using a photometer (BioTek, Gene Company Ltd., USA). Each sample was tested in triplicate. The samples were considered positive if the mean OD value was higher than the positive cut-off value.

\section{Results}

\section{Sand fly species identification}

Thirty specimens were identified by morphology as $\mathrm{Ph}$. chinensis. All other samples used in this study were identified as $P h$. chinensis by mtDNA cyt $b$ gene sequencing, which was conserved with the HM747267 sequence in GenBank database. The Ph. chinensis is the absolutely dominant sand fly species in the extension region of the Loess Plateau China.

\section{Blood sources identification}

The blood sources of the fed sand flies was according to the mtDNA cyt $b$ fragment of the animal species (Supplementary Fig. 1), include human (Homo sapiens, MT735217), pig (Sus domesticus, MT735219), chicken (Gallus gallus, MT735216), goat (Capra hircus, MT735218), dog (Canis familiaris, MT735215) and cattle (Bos taurus, MT735214). In SX, chicken blood was positive in all 12 pooled samples (100\%), followed by cattle (66.7\%), human (33.3\%), dog (33.3\%) and pig $(16.7 \%)$, whereas goat was negative in all samples. In YQ, chicken and human were the most common blood sources. Chicken blood was identified in $88.9 \%$ of pooled samples and $59.3 \%$ of individuals, and human blood was positive in $66.7 \%$ pooled samples and $26.3 \%$ individuals. In WX, goat blood was found in all pooled samples (100\%) and $34.3 \%$ individuals; human blood was detected in $70.0 \%$ pooled samples and $64.8 \%$ individuals. Chicken blood was positive in $10.0 \%$ pooled samples and 
$21.3 \%$ individuals. Dog blood was positive in 10.0\% pooled samples and $0.9 \%$ individuals (Table 3 ). In general, the sand fly has a wide range of blood sources in the extension region of the Loess Plateau, China.

Multiple blood sources within a sand fly were found in 54 individuals. Among them, 17 individual samples collected from YQ (14.4\%) with human plus chicken blood sources. The other 37 individual sand flies were collected from WX (34.3\%), including chicken + human $(n=10)$, goat + human $(n=24)$, chicken + goat $(n=1)$, chicken + goat + human $(n=1)$ and dog + goat + human $(n=1)$.

\section{Leishmania infection in sand flies}

Leishmania ITS 1 product were positive in five pooled samples $(5 / 59,8.5 \%)$ and two individual specimens (2/ 108, 1.9\%) from YQ population (Supplementary Fig. 2). The minimum infection rate of Leishmania in the sand fly population of YQ was 1.00\% (7/698). All sequences of Leishmania ITS 1 amplicons (MH200624) were conserved with $L$. donovani complex (L. donovani/L. infantum) [24]. Of the two positive individual specimens, one had human blood meal, and the other had chicken blood meal. No sample of SX and WX populations was positive in Leishmania DNA detection.

\section{Detection of Leishmania specific antibodies in dog sera}

Out of the 67 dog sera samples, 4 were identified as positive $(5.97 \%)$ in the detection of Leishmania specific antibodies according to the cut-off value using Dogs Leishmania Ab ELISA. The four positive dogs had no obvious pathological manifestations such as hair removal, desquamation, mental wilting, etc.

\section{Comprehensive detections from Yangquan}

Comprehensive results were obtained in the samples collected from Yangquan, Shanxi Province, China, which were summarized as below: i) A total of 3599 sand flies were collected, and Ph. chinensis was absolutely dominant species. ii) Chicken and human were the most common blood sources, and multiple blood sources within a sand fly were observed. iii) Leishmania DNA was detected in five pooled samples and two individual specimens, which sequences were conserved with $L$. donovani complex (L. donovani/L. infantum). iv) The positive rate of Leishmania specific antibodies in dog sera was $5.97 \%(4 / 67)$.

\section{Discussion}

Recent VL cases along the extension region of the Loess Plateau have been of the MT-ZVL type and have occurred mostly in children less than ten years old $[2,3]$. The primary vector of MT-ZVL is Ph. chinensis, which feeds on various animals as well as humans. We have verified dogs as a reservoir host of Leishmania; it may be the principal reservoir and the key to human VL cases in the region. A high Leishmania infection rate has been detected in dogs in the past, and human VL cases have been reduced after the elimination and suppression of local infected dogs $[11,25,26]$. Recently, VL cases in extension region of the Loess Plateau, China reappear after the disease eradication for 20 years, which posed a challenge for control.

Many sand fly species are known to rely on a broad range of species for blood meals [19, 20, 27-29], typically mammals [20, 30-33] but also various cold-blooded animals [21]. In this study, we investigated the blood sources of sand flies by amplified specific animal's mtDNA cyt $b$ fragments using PCR. The results showed that the chickens and humans were the most common blood sources of sand flies in the extension region of the Loess Plateau, China, while dogs, goats, cattle and pigs were also blood sources. In Jiuzhaigou of Sichuan, China, swine was the dominant blood source of sand flies, followed by chickens and dogs [22]. The results do not support the generally accepted opinion that humans and dogs are preferred feeding host of Ph. chinensis. As a good and sufficient blood source in the region, chickens may contribute to the sustainability of a large sand fly population, which was similar to other investigations $[34,35]$. Although chickens attract sand flies in the extension region of the Loess Plateau, China and in Brazil [35], the role of chickens in the epidemiology of the sand fly-borne diseases has not been defined yet. In this study, the proportions of blood sources were different among the three collection sites. The reason for this difference

Table 3 Blood sources detection results of the fed sandflies in the extension region of the Loess Plateau, China

\begin{tabular}{|c|c|c|c|c|c|c|c|}
\hline \multirow{2}{*}{$\begin{array}{l}\text { Collection } \\
\text { site }\end{array}$} & \multirow[t]{2}{*}{ Samples $(n)$} & \multicolumn{6}{|c|}{ Blood sources, number of positive samples (\%) } \\
\hline & & Human & Pig & Cattle & Dog & Chicken & Goat \\
\hline SX & Pooled samples (12) & $4(33.3 \%)$ & $2(16.7 \%)$ & $8(66.7 \%)$ & $4(33.3 \%)$ & $12(100 \%)$ & $0(0.0 \%)$ \\
\hline \multirow[t]{2}{*}{ YQ } & Pooled samples (9) & $6(66.7 \%)$ & $0(0.0 \%)$ & $0(0.0 \%)$ & $0(0.0 \%)$ & $8(88.9 \%)$ & $0(0.0 \%)$ \\
\hline & Individual samples (118) & $31(26.3 \%)$ & $0(0.0 \%)$ & $0(0.0 \%)$ & $0(0.0 \%)$ & $70(59.3 \%)$ & $1(0.8 \%)$ \\
\hline \multirow[t]{2}{*}{ WX } & Pooled samples (10) & $7(70.0 \%)$ & $0(0.0 \%)$ & $0(0.0 \%)$ & $1(10.0 \%)$ & $1(10.0 \%)$ & $10(100 \%)$ \\
\hline & Individual samples (108) & $70(64.8 \%)$ & $0(0.0 \%)$ & $0(0.0 \%)$ & $1(0.9 \%)$ & $23(21.3 \%)$ & $37(34.3 \%)$ \\
\hline
\end{tabular}

SX, Shanxian; YQ, Yangquan; WX, Wuxiang 
seems to be related to the blood sources available around the sandflies, depending on the environment.

We verified dogs as a reservoir host using samples from Yangquan, but in this region, unlike in Shanxian and Wuxiang, we did not detect dogs as a blood meal source of sand flies. However, Yangquan sand fly collection sites were far from areas frequented by dogs, and in the other regions, where the ecologic niches of sand flies were similar to that in Yangquan, dog was detected as a blood meal source. Thus we believe that dogs are likely a blood source for sand flies in Yangquan, and dogs are commonly seen in sand fly habitats there. Nonetheless, these data suggest caution in drawing final conclusions about the host-vector dynamics leading to VL infections in this region. Indeed, Leishmania spp. have been detected by PCR assay, such as in bovines (5\%), buffaloes (4\%), goats (16\%) [36], and even desert lizards [37]. In fact, goats are believed to constitute a reservoir host of L. donovani in Nepal [7]. Therefore, other reservoir animal hosts of Leishmania should be further investigated. Consequently, we believed that dogs were still the blood source of sand flies in Yangquan, which frequently moving around sand flies' habitats. In the next step, the sample size of the sandflies for blood sources analysis should be expanded. Multiple blood sources were found in individual fed specimens, suggesting a complex feeding behavior, which was critical for sand flies to transmit zoonotic diseases to humans.

In China, vector sand flies with Leishmania infection have been reported in Sichuan and Shaanxi provinces $[16,38]$. The natural infection rate of a new haplotype of L. donovani was $1.98 \%$ in some villages in Sichuan Province [39], consistent with the results of sand fly infection rate (minimum infection rate $1.00 \%$ ) in this study. To the best of our knowledge, this study was the first report of sand flies infected with Leishmania spp. in the extension region of the Loess Plateau, China. Monitoring natural Leishmania infection in sand flies provides critical information to assess the local epidemic risk of VL situation. Of the seven positive samples, the amplified sequences were all conserved with $L$. donovani complex (L. donovani/L. infantum). Multiple lines of evidence suggested that there were heterogeneous Leishmania strains in China. These strains were distinct from but phylogenetically related to $L$. donovani/L. infantum complex $[24,26,37,39,40]$. In this study, part of the ITS1 fragment sequence was amplified for Leishmania detection, which was not sufficient for typing the Leishmania. It would be interesting to isolate the local Leishmania species and determine its type using more variable regions of the genome.

Dogs were confirmed as the reservoir host of Leishmania spp. in mountainous type of zoonotic VL in the extension region of Loess Plateau, China [2, 3]. It has been reported that the positive rate of Leishmania spp. in dogs was above $50 \%$ in Jiuzhaigou of Sichuan, $41.9 \%$ in Heishui of Sichuan, and 77.21\% in Wenxian of Gansu, China [11, 25, 41]. In Shanxi Province, there was only one investigation in 1959, in which the Leishmania positive rate in dogs was $0.01 \%$ [16]. In this study, the antibodies positive rate in dog sera from Yangquan was 5.97\%. Among the seven positive sand fly samples tested, at least three contained human blood. Based on the preceding investigation, we believed that the screening and treatment of the disease sources need to be strengthened including asymptomatic infected persons and dogs.

Although we have detected the blood sources, Leishmania infection and the antibody of Leishmania in dog sera, there were still some limitations in this study. First, the primers for PCR detection of blood sources were designed based on the observation of the environment of the collection sites, but some blood sources animals may be missed because they are not observed. Moreover, the pooled samples were used for blood sources and Leishmania infection detection, making the positive rates not accurate. In addition, the investigation of potential reservoir hosts was insufficient in the study. Besides the dogs, other animals may serve as reservoir host. And the best way to verify Leishmania infection was to visualize the parasite under a microscope or isolate the culture from the samples. In the future, we will collect more animal blood samples to detect Leishmania infection and antibodies, and try to isolate the parasite from the samples, so as to provide more accurate guidance to prevent and control of VL in the extension region of Loess Plateau in China.

\section{Conclusions}

This study detected the blood sources and Leishmania parasites infection of sand flies by molecular methods in the extension region of Loess Plateau, China. The following comprehensive information indicates that the current risk of leishmaniasis endemic is high: high density of $P h$. chinensis vector, the positive infection of Leishmania in sand flies, the diverse blood sources of sand flies including humans and dogs, and positive antibody of Leishmania in local dog sera. Given the recent increase of VL cases, asymptomatic patients, dogs and other potential infected animals should be screened and treated. Furthermore, the density of sand flies needs to be controlled and personal protection should be strengthened.

\section{Supplementary information}

Supplementary information accompanies this paper at https://doi.org/10. 1186/s40249-020-00746-8.

Additional file 1: Supplementary Fig. 1 Agarose gel electrophoresis of PCR products for the detection of sandfly blood sources. A, chicken mtDNA cyt b gene amplification, lane 1, DNA marker; lane 2-14, 
detection samples. B, human mtDNA cyt $b$ gene amplification, lane 1 , DNA marker; lane 2-13, detection samples. C, goat mtDNA cyt $b$ gene amplification, lane 1, DNA marker; lane 2-15, detection samples

Additional file 2 Supplementary Fig. 2 Agarose gel electrophoresis of PCR products for Leishmania ITS1 detection in sand flies. Lane 1, positive control; lane 3-12, detection samples; lane 13, negative control. Lane 7,9 and 11 were positve samples

\section{Abbreviations}

DNA: Deoxyribonucleic acid; VL: Visceral leishmaniasis; PCR: Polymerase Chain Reaction; ELISA: Enzyme Linked Immunosorbent Assay; cyt b: Mitochondrial cytochrome b; ITS1: Internal transcribed spacer 1; YQ: Yangquan; WX: Wuxiang; SX: Shanxian

\section{Acknowledgments}

We are grateful to Hongwei Zhang, Chunli Su (Henan); Jingxia Chen, Junying Zhao, Peifang Dai, Xiaodong Tian (Shanxi) and Zhenzhou Yang, who provided field assistance in the study.

\section{Authors' contributions}

All authors contributed to the collection of sand flies. YM and HP conceived and designed the experiments. HYC and YM identified specimens by morphological characters. HMC and HYC performed PCR assay. HP did ELISA test. FT, HP and YM did data analysis and wrote manuscript. The author(s) read and approved the final manuscript.

\section{Funding}

This work was supported by National Natural Sciences Foundation of China (No. 31970445 \& 81371848), and Shanxi Province Science and Technology Department (201803D31205).

\section{Availability of data and materials}

The datasets used and/or analyzed during the current study are available from the corresponding author on reasonable request.

\section{Ethics approval and consent to participate}

This study was carried out in strict accordance with the National Natural Science Foundation of China ethical guidelines for biomedical research involving living animals and human subjects. The owners of the dogs had been informed in advance and consented to the blood collection.

\section{Consent for publication}

Not applicable.

\section{Competing interests}

The authors declare that they have no competing interests.

\section{Author details}

'Department of Naval Medicine, Naval Medical University, 800 Xiangyin Road, Shanghai 200433, China. ${ }^{2}$ Institute of Disease Control and Prevention of People's Liberation Army of China, Beijing 100071, China. ${ }^{3}$ Department of Medical Microbiology and Parasitology, College of Basic Medical Sciences, Naval Medical University, 800 Xiangyin Road, Shanghai 200433, China.

Received: 26 May 2020 Accepted: 26 August 2020

Published online: 31 August 2020

\section{References}

1. Guan LR. Present situation of visceral leishmaniasis and prospect for its control in China. Zhongguo Ji Sheng Chong Xue Yu Ji Sheng Chong Bing Za Zhi. 2009;27(5):394-7.

2. Wang JY, Cui G, Chen HT, Zhou XN, Gao CH, Yang YT. Current epidemiological profile and features of visceral leishmaniasis in people's Republic of China. Parasit Vectors. 2012;5:31

3. Nzelu CO, Caceres AG, Arrunategui-Jimenez MJ, Lanas-Rosas MF, YanezTrujillano HH, Luna-Caipo DV, et al. DNA barcoding for identification of sand fly species (Diptera: Psychodidae) from leishmaniasis-endemic areas of Peru. Acta Trop. 2015;145:45-51.
4. Guan LR, Wu ZX. Historical experience in the elimination of visceral leishmaniasis in the plain region of eastern and Central China. Infect Dis Poverty. 2014;3(1):10

5. Zhao S, Li Z, Zhou S, Zheng C, Ma H. Epidemiological feature of visceral leishmaniasis in China, 2004-2012. Iran J Public Health. 2015;44(1):51-9.

6. Zhou ZB, Li YY, Zhang Y, Li SZ. Prevalence of visceral leishmaniaisis in China in 2018. Chin J Parasit Dis. 2020;38(2):175-80 87.

7. Lun ZR, Wu MS, Chen YF, Wang JY, Zhou XN, Liao LF, et al. Visceral leishmaniasis in China: an endemic disease under control. Clin Microbiol Rev. 2015;28(4):987-1004

8. Wang ZJ, Xiong GH, Guan LR. Achievement on the epidemiology and control of kala-azar in China. Chin J Epidemiol. 2000;21:51-4.

9. Guan LR, Qu JQ, Chai JJ. Leishmaniasis in China-present status of prevalence and suggestions on its control. Endem Dis Bull. 2000;15:49-52.

10. Xiong GH. Present epidemiological situation of visceral leishmaniasis in China. Endem Dis Bull. 1992;7:113-25.

11. Li YF, Zhong WX, Zhao GH, Wang HF. Prevalence and control of kala-azar in China. J Pathog Biol. 2011;6:629-31.

12. Province $\mathrm{HaFPCOH}$. 2018. Available from: http://www.hnwsjsw.gov.cn/ channels/109.shtml.

13. Yang $S L$, Song $L$, Ma GQ, Wang BZ, Wang CH, Su LJ. Investigation of 4 cases of kala-azar in Anyang City, Henan Province. China Chin Endemiol. 2018:37(12):1027.

14. Pereira Junior AM, Souza ABN, Castro TS, da Silva MS, de Paulo PFM, Ferreira GEM, et al. Diversity, natural infection and blood meal sources of phlebotomine sandflies (Diptera, Psychodidae) in the western Brazilian Amazon. Mem Inst Oswaldo Cruz. 2019;114:e190170.

15. Pereira Junior AM, Teles CB, de Azevedo dos Santos AP, de Souza RM, Marialva EF, Pessoa FA, et al. Ecological aspects and molecular detection of Leishmania DNA Ross (Kinetoplastida: Trypanosomatidae) in phlebotomine sandflies (Diptera: Psychodidae) in terra firme and varzea environments in the middle Solimoes region, Amazonas state. Brazil Parasit Vectors. 2015;8:180.

16. Xiong GH, Jin CF, Guan LR. Chinese Sandflies. Beijing: Science China Press; 2016.

17. Chen HM, Chen HY, Gao JP, Li KL, Yang ZZ, Peng H, et al. Ecological niches of sandfly (Diptera: Psychodidae) in the extension region of loess plateau, China: an endemic focus of visceral leishmaniasis. Chin J Vector Biol Control. 2019:30(6):597-602.

18. Esseghir S, Ready PD, Ben-Ismail R. Speciation of Phlebotomus sandflies of the subgenus Larroussius coincided with the late Miocene-Pliocene aridification of the Mediterranean subregion. Biol J Linn Soc. 2000;70(2):189219.

19. Abbasi I, Cunio R, Warburg A. Identification of blood meals imbibed by phlebotomine sand flies using cytochrome b PCR and reverse line blotting. Vector Borne Zoonotic Dis. 2009;9(1):79-86.

20. Garlapati RB, Abbasi I, Warburg A, Poche D, Poche R. Identification of bloodmeals in wild caught blood fed Phlebotomus argentipes (Diptera: Psychodidae) using cytochrome b PCR and reverse line blotting in Bihar. India J Med Entomol. 2012:49(3):515-21.

21. Maia C, Parreira R, Cristovao JM, Freitas FB, Afonso MO, Campino L. Molecular detection of Leishmania DNA and identification of blood meals in wild caught phlebotomine sand flies (Diptera: Psychodidae) from southern Portugal. Parasit Vectors. 2015:8:173.

22. Chen H, Li K, Shi H, Zhang Y, Ha Y, Wang Y, et al. Ecological niches and blood sources of sand fly in an endemic focus of visceral leishmaniasis in Jiuzhaigou, Sichuan. China Infect Dis Poverty. 2016;5:33.

23. Schonian G, Nasereddin A, Dinse N, Schweynoch C, Schallig HD, Presber W, et al. PCR diagnosis and characterization of Leishmania in local and imported clinical samples. Diagn Microbiol Infect Dis. 2003;47(1):349-58.

24. Yang BB, Guo XG, Hu XS, Zhang JG, Liao L, Chen DL, et al. Species discrimination and phylogenetic inference of 17 Chinese Leishmania isolates based on internal transcribed spacer 1 (ITS1) sequences. Parasitol Res. 2010;107(5):1049-65.

25. Zhao GH, Yin K, Zhong WX, Xiao T, Wei QK, Cui Y, et al. Epidemiological investigation of asymptomatic dogs with Leishmania infection in southwestern China where visceral leishmaniasis is intractable. Korean J Parasitol. 2016;54(6):797-801

26. Sun K, Guan W, Zhang JG, Wang YJ, Tian Y, Liao L, et al. Prevalence of canine leishmaniasis in Beichuan County, Sichuan, China and phylogenetic evidence for an undescribed Leishmania sp. in China based on 7SL RNA. Parasit vectors. 2012:5:75 
27. Li K, Chen H, Jiang J, Li X, Xu J, Ma Y. Diversity of bacteriome associated with Phlebotomus chinensis (Diptera: Psychodidae) sand flies in two wild populations from China. Sci Rep. 2016;6:36406.

28. Ready PD. Biology of phlebotomine sand flies as vectors of disease agents. Annu Rev Entomol. 2013;58:227-50.

29. Warburg A, Faiman R. Research priorities for the control of phlebotomine sand flies. J Vector Ecol. 2011;36(Suppl 1):S10-6.

30. Kent RJ, Norris DE. Identification of mammalian blood meals in mosquitoes by a multiplexed polymerase chain reaction targeting cytochrome B. Am J Trop Med Hyg. 2005;73(2):336-42.

31. Jimenez M, Gonzalez E, Iriso A, Marco E, Alegret A, Fuster F, et al. Detection of Leishmania infantum and identification of blood meals in Phlebotomus perniciosus from a focus of human leishmaniasis in Madrid. Spain Parasitol Res. 2013;112(7):2453-9.

32. Pruzinova K, Sadlova J, Seblova V, Homola M, Votypka J, Volf P. Comparison of bloodmeal digestion and the peritrophic matrix in four sand fly species differing in susceptibility to Leishmania donovani. PLoS One. 2015;10(6): e0128203.

33. Gebresilassie A, Abbasi I, Aklilu E, Yared S, Kirstein OD, Moncaz A, et al. Hostfeeding preference of Phlebotomus orientalis (Diptera: Psychodidae) in an endemic focus of visceral leishmaniasis in northern Ethiopia. Parasit Vectors. 2015:8:270.

34. de Avila MM, Brilhante AF, de Souza CF, Bevilacqua PD, Galati EAB, Brazil RP. Ecology, feeding and natural infection by Leishmania spp. of phlebotomine sand flies in an area of high incidence of American tegumentary leishmaniasis in the municipality of Rio Branco, Acre, Brazil. Parasit Vectors. 2018;11(1):64.

35. Alexander B, de Carvalho RL, McCallum H, Pereira MH. Role of the domestic chicken (Gallus gallus) in the epidemiology of urban visceral leishmaniasis in Brazil. Emerg Infect Dis. 2002;8(12):1480-5.

36. Bhattarai NR, Van der Auwera G, Rijal S, Picado A, Speybroeck N, Khanal B, et al. Domestic animals and epidemiology of visceral leishmaniasis. Nepal Emerg Infect Dis. 2010;16(2):231-7.

37. Zhang JR, Guo XG, Liu JL, Zhou TH, Gong X, Chen DL, et al. Molecular detection, identification and phylogenetic inference of Leishmania spp. in some desert lizards from Northwest China by using internal transcribed spacer 1 (ITS1) sequences. Acta Trop. 2016;162:83-94.

38. Li XY, Zhang Z, Liu DL, Wang FP, Wang AL, Wang TH, et al. Identification of Leishmania in Shaanxi Province based on repearted DNA sequence. Zhongguo Ji Sheng Chong Xue Yu Ji Sheng Chong Bing Za Zhi. 2018;36(3): 271-4.

39. Wei F, Shang L, Jin H, Lian H, Liu W, Li Z, et al. Molecular detection and genetic diversity of Leishmania donovani in naturally infected Phlebotomus chinensi from southwestern China. Vector Borne Zoonotic Dis. 2011;11(7): $849-52$

40. Yang BB, Chen DL, Chen JP, Liao L, Hu XS, Xu JN. Analysis of kinetoplast cytochrome b gene of 16 Leishmania isolates from different foci of China: different species of Leishmania in China and their phylogenetic inference. Parasit Vectors. 2013;6:32.

41. Wang JY, Chen SB, Gao CH, Jin CF, Feng Y, Zhang CJ, et al. Servey on the Leishmania infantum asyptomatic infection in dogs in Wenxian county of Gansu province. Chin J Zoonoses. 2006;22(8):9.

Ready to submit your research? Choose BMC and benefit from:

- fast, convenient online submission

- thorough peer review by experienced researchers in your field

- rapid publication on acceptance

- support for research data, including large and complex data types

- gold Open Access which fosters wider collaboration and increased citations

- maximum visibility for your research: over $100 \mathrm{M}$ website views per year

At BMC, research is always in progress.

Learn more biomedcentral.com/submissions 